\title{
Intersatellite Links:
}

\section{Lower Layer Protocols for Autonomous Constellations}

\author{
Kerri L. Kusza, Michael A. Paluszek \\ Princeton Satellite Systems \\ 333 Witherspoon Street \\ Princeton, NJ 08540 \\ 609-279-9606 \\ kusza@stanford.edu,map@psatellite.com
}

\begin{abstract}
In order to have autonomous formation-flying satellite constellations in low earth orbit (LEO), the satellites in the constellation must be able to communicate with each other directly via intersatellite links (ISLs). Current ISL implementations use lower layer link protocols based on existing networking protocols such as X.25 and LAP-B, which were not designed specifically for ISL use. This paper compares current and upcoming lower layer protocols in an attempt to identify a protocol for widespread ISL use such that interaction among different constellations is possible and the addition of new satellites to existing constellations is simpler.
\end{abstract}

\subsection{INTRODUCTION}

Recent advances in micro electro-mechanical systems (MEMS) permit robust microsatellites to be built. The combined resources of several of these smaller, smarter satellites for applications such as distributed aperture remote sensing, has significant scientific, performance and cost advantages over using large, heavy, single-mission satellites. In order to effectively combine the resources of autonomous, formationflying constellations of smaller satellites, the satellites must have the ability to communicate with each other.

Autonomy implies minimal dependence on ground stations for communication purposes, and so intersatellite links (ISLs) must be used to allow satellites to share their individual information and use their combined resources to achieve a more complex goal. Selecting the lower layer protocols for use with an ISL-based communication system demands a comparison of system requirements against the functionality of existing standards. Using an existing standard is simplest in terms of cost and time. However, standards currently used in similar applications for ISLs are based on terrestrial networking protocols developed over a decade ago and are not necessarily optimized for short range space links. Modifications to optimize existing protocols for use in ISLs are not simple and are almost always proprietary. This does not encourage communication with other nearby constellations or simplify adding new satellites to an existing constellation.

This work was supported by the U.S. Department of Defense and the U.S. Air Force Office of Scientific Research under Contract F29601-99-C-0029.

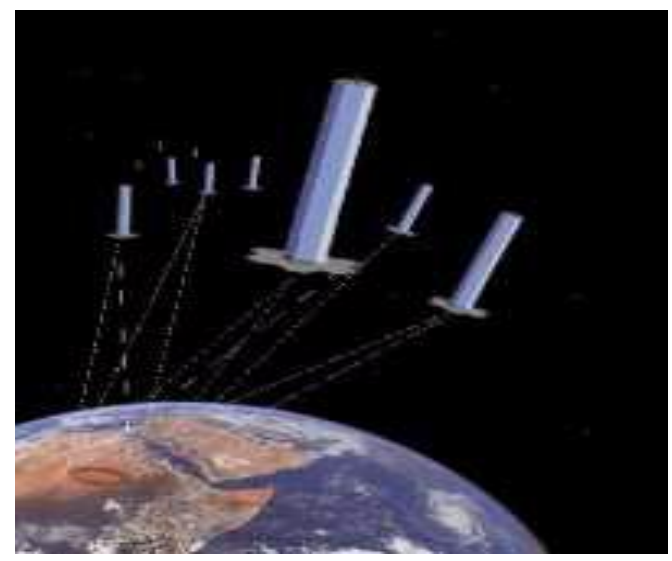

Figure 1: Conceptual depiction of autonomous constellation courtesy of the AFRL TechSat 21 (Technology Satellite of the 21st Century) program.

This paper compares and contrasts existing standards such as X.25/LAP-B, TCP/IP, ATM, and even the wireless IEEE 802.11 protocol to determine which best meets the needs of the ISL lower layers for an autonomous constellation. The comparison also includes a discussion of the upcoming Consultative Committee for Space Data Systems (CCSDS) Proximity-1 protocol that was created specifically for proximityrange space links, and evaluates the CCSDS Proximity-1 stack against the X.25 stack.

\section{ISL and Low Layer Protocol Definition}

Intersatellite links are two-way communication paths between satellites. They have the potential to provide flexibility in the space segment implementation while maintaining or reducing the cost of the system's earth segment [1]. As described in Section 2, the low layer protocol choice is a key part of ISL design for autonomous constellations because it must guarantee reliable transfer. Reliable transfer is critical for autonomous constellations from a navigation and data collection standpoint. Messages must be delivered error-free, in order, no duplicates, and without added delay[2]. Additional requirements, including data rate, range and power, must also be considered. Autonomous constellations in general also require significant networking and multiple access capability. 


\section{Types of ISL Media and Data Rate Requirements}

Radio frequency (RF) and Optical (laser) are the two primary communication media for an ISL. Optical has the advantage of higher data rates, low probability of intercept, smaller size, and lower power. However, it also has much more complex acquisition and tracking, may have additional delays in converting electrical signals to optical, and is fairly new as far as flight implementation is concerned. The current advantage is with radio links for throughputs on the order of 10Mbps. Optical links may be more advantageous for throughputs at several tens of Mbps or more [3]. For the requirements of an autonomous constellation of LEO microsatellites or nanosatellites with data rates currently on the order of $1 \mathrm{Mbps}$, RF links are more than adequate.

\section{ISL Multiple Access}

Multiple access schemes require an additional layer in the low level stack for media access control (MAC) which is discussed in the brief link layer outline in Section 2. Use of a spread spectrum link for multiple access in an autonomous constellation is desirable as spread spectrum links can provide resistance to intentional jamming, mask the transmitted signal in the background noise to prevent eavesdropping, provide resistance to degrading and multipath effects on the signal, and also provide range-measuring capability. The two major types of spread-spectrum systems are direct-sequence spread spectrum (DSSS) and frequency-hop spread spectrum (FHSS). In DSSS, a spreading code with a rate much higher than the data rate multiplies the data sequence to spread the spectrum, and for FHSS, a synthesizer driven by a pseudorandom noise generator provides a carrier that changes frequency in a pseudorandom manner [4].

Using the IEEE 802.11 standard as a reference (with US standards requiring $<1 \mathrm{~W}$ of RF power), current implementations of FHSS achieve rates only up to $1-2 \mathrm{Mbps}$. Current implementations of DSSS can achieve rates up to $11 \mathrm{Mbps}$ [5]. FHSS will get faster when the cost of using an equalization circuit to reduce inter-symbol interference (ISI) at higher data rates goes down. FHSS should be used where it is desirable to avoid high power, narrow band interference and the lower data rate of a few Mbps is acceptable [3]. Section 3 will discuss IEEE 802.11 in more detail.

\section{ISL Constellations and Low Layer Protocols}

When considering ISL low layer protocols for autonomous constellations, it is useful to briefly discuss existing nonautonomous satellite constellations that have successfully used crosslinks.

Milstar was the first geostationary (GEO) constellation to use intersatellite links and onboard processing to get a short message to strategic bomber pilots and missile commanders during a nuclear war. The original spacecraft were initially large and consumed a lot of power, but new designs are sig- nificantly smaller and more efficient. The first Milstar launch was in 1994. The first commercial test of onboard processing and intersatellite links was Iridium (LEO) in 1998.



Figure 2: An artist's conception of TDRSS.

Another well-known constellation that uses crosslinks is NASA's TDRSS (Tracking and Data Relay Satellite System). TDRSS, also GEO, tracks and communicates with Earth-orbiting spacecraft such as the International Space Station (ISS) and the Hubble Space Telescope (HST) and transmits their data to ground stations on Earth. It offers both single access and multiple access support in downlinking data and can handle a variety of frequency bands, but the system is not very similar to an autonomous, formation-flying constellation in terms of data rate, power consumption, or size.

Out of the multitude of commercial satellites currently on orbit or the design table, only a handful have ISLs as opposed to a bent-pipe or demod/remod approach. Within that handful, some are GEO constellations, some are LEO constellations and a few are in-between and will be referred to as MEO (medium earth orbit) constellations [6].

Most of the broadband constellations such as Spaceway (Hughes) and V-Stream (PanAmSat) are GEO and in general have power, mass, and data rate requirements that exceed the range currently required by an autonomous LEO constellation. The MEO and LEO constellations have more similar requirements and some relevant commercial constellations are listed in Table 1. Most of these constellations do not use ISLs, and those that do are further detailed in Table 2.

It is interesting to note that out of the fixed constellations that use ISLs, only Iridium has actually made it to launch and operation. 
TABLE 1. Constellations in LEO/MEO

\begin{tabular}{|c|c|c|c|c|c|}
\hline Constellation & $\begin{array}{c}\text { Corporate } \\
\text { Sponsor }\end{array}$ & $\begin{array}{l}\text { Orbit, Altitude, } \\
\text { Number of Sats }\end{array}$ & Mass & ISL & Operation \\
\hline Ellipso & $\begin{array}{l}\text { Boeing, Lockheed, } \\
\text { L3 Comm, Harris }\end{array}$ & $\begin{array}{c}\text { MEO, 7000km } 17 \text { sats } \\
\text { elliptical, equatorial }\end{array}$ & $500 \mathrm{~kg}$ & No & $2002 *$ \\
\hline Globalstar & $\begin{array}{l}\text { Qualcomm, Alca- } \\
\text { tel }\end{array}$ & LEO, $1410 \mathrm{~km}, 48$ sats & $450 \mathrm{~kg}$ & No & 1999 \\
\hline Iridium & Motorola & LEO, $780 \mathrm{~km}, 66$ sats & $700 \mathrm{~kg}$ & Yes & 1998 \\
\hline Leo One & $\begin{array}{l}\text { DaimlerChrysler } \\
\text { Aero, Lockheed }\end{array}$ & LEO, $950 \mathrm{~km}, 48$ sats & $192 \mathrm{~kg}$ & No & $2003 *$ \\
\hline Orblink & Orbital Sciences & MEO, $9000 \mathrm{~km}, 7$ sats & $1360 \mathrm{~kg}$ & Yes & $2002 *$ \\
\hline Skybridge & Alcatel & LEO, $1500 \mathrm{~km}, 80$ sats & $1250 \mathrm{~kg}$ & No & $2002 *$ \\
\hline Teledesic & $\begin{array}{l}\text { ICO, Motorola, } \\
\text { Lockheed, Boeing }\end{array}$ & LEO, $700 \mathrm{~km}, 288 ?$ sats & $771 \mathrm{~kg}$ & Yes & $2005^{*}$ \\
\hline
\end{tabular}

TABLE 2. Constellations with ISLs

\begin{tabular}{|c|c|c|c|c|c|}
\hline Constellation & $\begin{array}{l}\text { ISL } \\
\text { type }\end{array}$ & ISL band & $\begin{array}{l}\text { ISL Data } \\
\text { Rate }\end{array}$ & $\begin{array}{l}\text { Connection } \\
\text { Description }\end{array}$ & $\begin{array}{c}\text { ISL } \\
\text { Protocols }\end{array}$ \\
\hline 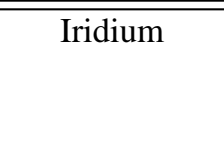 & 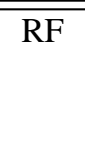 & $\begin{array}{c}22.55- \\
23.55 \mathrm{GHz}\end{array}$ & $25 \mathrm{Mbps}$ & $\begin{array}{l}4 \text { per satellite } \\
2 \text { intra-plane } \\
2 \text { inter-plane }\end{array}$ & $\begin{array}{l}\text { Motorola proprietary } \\
\text { ATM-like switching }\end{array}$ \\
\hline Orblink & $\mathrm{RF}$ & $\begin{array}{c}65.0- \\
71.0 \mathrm{GHz}\end{array}$ & 15 Gbps & $\begin{array}{l}2 \text { per satellite } \\
2 \text { intra-plane }\end{array}$ & $\begin{array}{l}\text { Proprietary simple } \\
\text { switching }\end{array}$ \\
\hline Teledesic & $\mathrm{RF}$ & $60 \mathrm{GHz}$ & $155 \mathrm{Mbps}$ & $\begin{array}{l}8 \text { per satellite } \\
\text { Permanent and } \\
\text { dynamic links }\end{array}$ & $\begin{array}{l}\text { Teledesic proprietary } \\
\text { ATM-like switching }\end{array}$ \\
\hline
\end{tabular}

\section{Other Formation-Flying Constellations}

Commercial LEO constellations are not the best comparison to an autonomous formation-flying constellation, but it is interesting to note that most do not use ISLs, and of those that do, it is clear from the above table that there is no standard non-proprietary protocol. However, programs do exist to develop autonomous, formation-flying constellations with ISLs, such as:

- NASA's New Millennium Program Space Technology 5 (NMP ST5) with RF ISLs.

- Surrey Satellite Technology (SSTL) just launched the (Surrey Nanosatellite Applications Platform) SNAP-1 and Tsinghua-1 with RF ISLs [6], [7].
- TechSat 21 (AFOSR) autonomous cluster of formationflying microsatellites that operate cooperatively to perform the function of a larger, single satellite. The satellites will share data processing, payload, and mission functions via RF ISLs.

The NMP ST5, SNAP-1, and TechSat 21 programs are discussed in detail in Section 4 because they use RF ISLs for formation-flying constellations and are similar enough to make a comparison of their lower layer protocol choices.

\subsection{BRIEF NETWORKING OVERVIEW}

This section considers standard lower layer protocols or protocol combinations for use with ISLs on an autonomous formation-flying constellation. A brief overview of the OSI 


\begin{tabular}{l}
$\begin{array}{l}\text { Application Layer } \\
\text { (Messages) }\end{array}$ \\
$\begin{array}{l}\text { Presentation } \\
\text { (Format of Data) }\end{array}$ \\
$\begin{array}{l}\text { Session } \\
\text { (Dialog Btwn Apps) }\end{array}$ \\
$\begin{array}{l}\text { Transport } \\
\text { (Segments) }\end{array}$ \\
$\begin{array}{l}\text { Network } \\
\text { (Packets, Datagrams) }\end{array}$ \\
\hline $\begin{array}{l}\text { Data Link } \\
\text { (Frames) }\end{array}$ \\
\hline $\begin{array}{l}\text { Physical Layer } \\
\text { (Bits) }\end{array}$ \\
\hline
\end{tabular}

Manages user interface to network. File access and transfer, virtual terminal. Application Programming

Format conversion, data encryption, compression and expansion.

Establishes, maintains and synchronizes dialog between communicating applications on remote computers.

Sequencing, acknowledgment, flow control. Message multiplexing. Fragmentation and reassembly.

Creates and routes packets (also called datagrams). Network-wide logical addressing.

Creates frames, encapsulates packets or data. Physical address management. Error checking / retransmission.

Transmits a bit stream that meets physical and electrical interface requirements between user and network

Figure 3: The OSI reference model. Although current communication networks do not explicitly follow this model, it is a good reference for understanding what is needed to make communication work. This paper is primarily concerned with the functions of the unshaded lower layers in this figure.

model and description of lower layer functionality is followed by a discussion of the protocols[10].

\section{The OSI Reference Model}

The ISO (International Standards Organization) created the OSI model to define a common way to connect processes. It is not necessarily a followed model in communication networks, but it serves well as a basic guide for what needs to happen in order for communication to be successful. The OSI model has seven layers and is outlined in Figure 3.

\section{Lower Layer Functionality}

The lower three layers of the OSI model, the network, data link, and physical layers, are of primary concern in this paper, since they have the largest role to play when it comes to reliable and efficient communication via ISL. Many of the standard protocols such as X.25 / LAP-B, ATM, TCP/IP, and IEEE 802.11 cover all or parts of these three layers within their protocol definitions, and the boundaries between these layers get blurred. It is simpler to group the three lower layers together when attempting to compare protocol stacks for ISLs. The next section briefly covers some important considerations of lower layer functionality such as connection-oriented vs. connectionless, error control, flow control, the role of the LLC (logical link control) and MAC sublayers, the space channel environment before going through the previously listed protocols in further detail. The X.25/LAP-B and IEEE 802.11 protocols are covered in more depth than ATM and TCP/IP as they are better fits for an autonomous formation-flying constellation.

\section{Logical Link Control and Media Access Control}

Logical link control (LLC) is a subclass of HDLC (high level data link control) ${ }^{1}$ that is often used as the link layer protocol in local area networks (LANs). LANs typically have relatively short, low BER links that operate at high bit rates. Errors are relatively infrequent and the round trip time (RTT) is fast. It is acceptable for these networks to operate in connectionless, best-try mode where all retransmission and flow control functions, if needed, are left to a higher protocol layer. LLC can be used whenever error detection, correction, and sequencing are either unnecessary or implemented by a higher layer and do not need to be replicated in the lower layers. LLC is used to initiate transfer with minimum overhead (each additional layer adds headers with bits in addition to the information to be transferred). If run in a connectionoriented mode instead, LLC is similar to HDLC except framing and error detection are done in the MAC sublayer. As shown in Figure 4, the MAC layer controls resource sharing, collision avoidance, and interface with the physical layer.

1. To help clarify, a connection-oriented LLC is most similar to IEEE 802.2, the IEEE version of HDLC. HDLC was originally called SDLC by IBM, and renamed HDLC when ISO made a standard out of it. There is also the ITU-T version of HDLC called link access protocol, or LAP, and balanced mode is LAPB. IEEE 802.3 is a MAC layer for bus networks, 802.4 is a MAC layer for token bus networks, and 802.5 is a MAC layer for token ring networks. IEEE 802.11 is the MAC and physical layers for a wireless network and is discussed in further detail later in this paper. 


\begin{tabular}{|c|}
\hline Data Link Layer \\
\hline Physical Layer \\
\hline
\end{tabular}

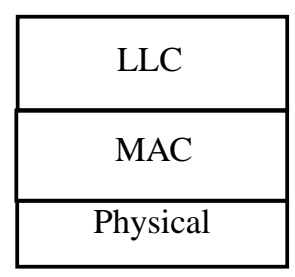

Figure 4a: In a low BER and high bit rate LAN such as an Ethernet, often the data link layer has LLC and MAC sublayers. The LLC is typically connectionless to avoid setup overhead, and the MAC ensures that the link is used fairly, as well as interfacing with the physical layer.

\section{Causes of Error in the Space Channel}

There are several different kinds of error that need to be considered and corrected for in order to ensure the desired BER and positioning accuracy specified for an autonomous constellation. The first kind are bit errors. Single and double bit errors are usually simple to correct for using CRC codes. However, burst errors, where many bits are corrupted at once, may not be corrected by CRC codes and occur more frequently than single bit errors. Depending on the burst length, FEC should be able to help with recovery and avoid retransmissions. Bit slips may occur, where bits are lost due to variations in the respective clock rates of the transmitter and receiver. There is also the possibility that an entire packet is lost due to incorrect addressing, or hardware error because of electrical interference or thermal noise. In this case, it is necessary to either retransmit or ignore the lost packet. The possibility of link failure, due to a damaged or out of range spacecraft, also must be designed for. Space link designs also have to consider variable RTTs, increased noise or bursts of noise, limited bandwidth, single event upsets, spacecraft antenna obscurations, limited processing power, program memory, and data buffering, and sometimes the forward and return links are not symmetric [1], [11].

\subsection{Existing Lower Layer Open Protocols}

\section{HDLC and X.25}

The high-level data link control (HDLC) protocol is designed for the data link layer, to perform synchronous or asynchronous, code-transparent transmission. It has been used primarily for higher bit rate, long range links such as ground-space satellite links or multiplexed circuit networks. The X.25 packet-switched network layer protocol runs on a data link layer

\begin{tabular}{|c|}
\hline Data Link \\
\hline MAC \\
\hline Physical Layer \\
\hline
\end{tabular}

Figure 4b: In a multiple access system such as a wireless network, a MAC layer is implemented to control shared access of the communications medium, collision avoidance between users, and to interface with the physical layer such as in IEEE 802.11.

called LAP-B (balanced link access procedure) that is based on the asynchronous balanced mode (ABM) of HDLC [12], [13].

In addition to ABM, there are two other modes of HDLC, normal response mode (NRM) and asynchronous response mode (ARM). NRM involves a master-slave relationship between users, the master commands and the slave(s) respond. ARM also uses a master-slave relationship, but the slaves are effectively allowed to talk without being spoken to first. ABM is the democratic process where each user has an equal status and may both command or respond. There are also three non-operational modes of HDLC that deal with disconnecting and initialization.

HDLC uses a flag to signal the start and end of a frame (01111110) and bit-stuffing to avoid a repeat of that sequence in the rest of the frame. HDLC uses continuous RQ with reject (go-back-n), selective reject, and multi-selective reject options. The information to be sent is encapsulated in a variable length frame called an I-frame. HDLC can also use the I-frame to piggyback acknowledgments in the other direction for its RQ functions. HDLC uses unnumbered frames (U-frames) to set up and tear down a link, and supervisory frames (S-frames) for error and flow control. Recall also that HDLC uses CRC-CCITT as a frame check sequence (FCS) for error checking. The send window of HDLC has been extended from 3 bits (can send 7 frames at a time) to 7 bits (can send 127) for long range links. In general, HDLC assumes a fairly reliable link and focuses more on flow control than error control.

LAP-B is used to control I-frames being sent across a packet-switched network, such as an X.25 network. As mentioned previously, LAP-B is HDLC ABM, and treats all Iframes as though they were command frames. LAP-B could not handle multiple physical links until the addition of the multilink procedure (MLP) extension. MLP treats a set of single link procedures as though they were a pool of links to transfer user frames over. It has its own sequence numbers, 


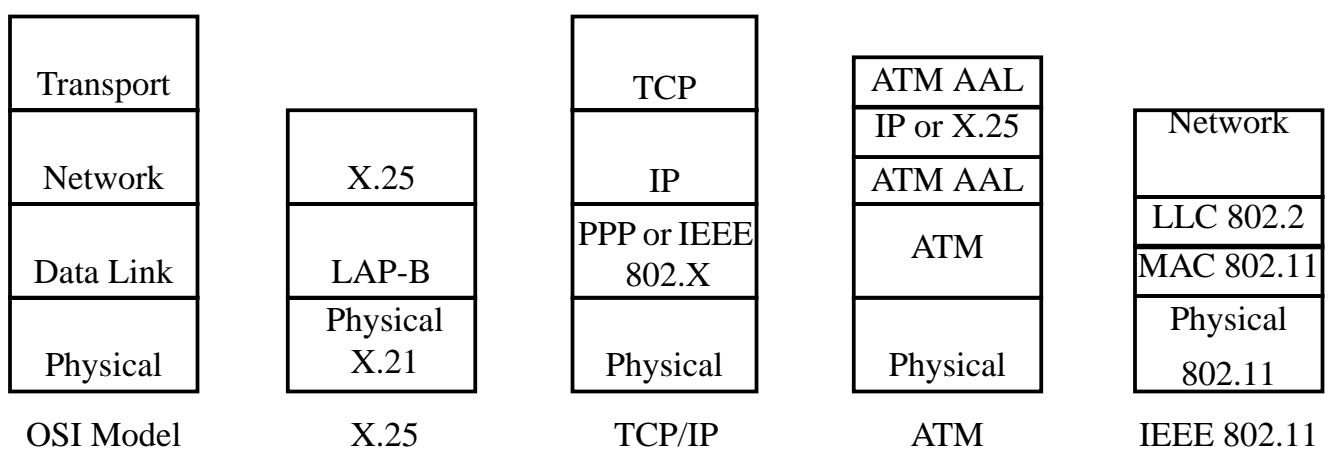

Figure5: Possible lower layer protocol stacks for an ISL, taken from the pool of existing commercial standards. IEEE 802.2 is the IEEE modified version of HDLC.

and if a link goes down, it will simply continue using the reduced set of links in its pool.

The ITU-T X.25 standard specifies X.21 at the physical layer and LAP-B at the data link layer. X.25 functions primarily at the network layer. The basic strategy behind X.25 is to allocate buffer space to a "virtual circuit" on initialization, then use the sliding window algorithm for flow control to keep the sender from overrunning the allocated buffers. The initial set up of the virtual circuit can be rejected by the sender if they know that there won't be enough buffers allocated to them. If this happens, or if a virtual circuit cannot be set up (due to heavy loading) then a clear-request control packet goes back to the sender, explaining why a connection could not be established.

X.25 and LAP-B can certainly work over intersatellite links for an autonomous formation-flying constellation, and there currently are versions of HDLC being flown on some constellations designed with ISLs (such as SNAP-1). The question is whether or not there will ever be a common implementation widely used enough that it will be standard when the goal is to have different kinds of satellites from different clusters or even missions easily interact, or whether a protocol designed specifically for intersatellite or wireless links would work better.

\section{$T C P / I P$}

TCP/IP is a two-protocol stack that has taken over 30 years to evolve. The network layer protocol is called the Internet Protocol (IP) and the transport layer is called Transport Control Protocol (TCP). The two protocols are fairly intuitive and public domain - there are no licensing fees for using them. The ISO has created standards based on them, however [14].

The primary purpose of the TCP/IP combination was to build an intercon-

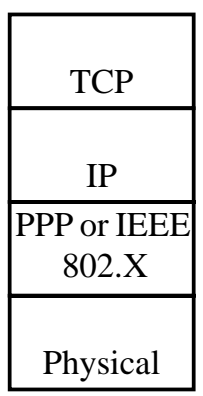

TCP/IP transfer. Another goal was not only to interconnect networks with the same or compatible architectures, but to connect networks that were physically different.

In TCP/IP the transport layer is providing the reliable end-toend data transfer, and TCP is connection-oriented even though its IP is connectionless. IP can run over X.25, ATM, IEEE 802.2 and a number of other lower layers. It has been said that IP can run over two tin cans and a piece of string [8].

Although the TCP/IP combination is in fact a reliable communication protocol stack, it is intended to connect and run over many different physical networks with their own existing lower layer protocols. Here it is not currently considered as a stand-alone candidate lower layer protocol stack for use in an autonomous constellation in this paper, but it may be considered at the internetworking level for a more advanced formation-flying constellation.

\section{ATM}

Asynchronous Transfer Mode (ATM), or cell-switching, is used primarily for broadband multiservice networks and services such as voice, images, data, video, and videoconferencing. ATM uses a packet transfer mode based on asynchronous time division multiplexing. User information is transported in fixed-length

\begin{tabular}{|c|}
\hline ATM AAL \\
\hline IP or X.25 \\
\hline ATM AAL \\
\hline ATM \\
\hline Physical \\
\hline
\end{tabular}

ATM blocks, called ATM cells. Each cell is 48 bytes long plus 5 bytes of header for a total of 53 bytes. A cell is a hybrid of digitized voice transmission slots and variable length, multiplexed data frames. It is much easier to implement switches and hardware for fixed length cells, since everything is uniform. No error control is performed on cells, and no sequence numbers are required for retransmission [8], [9]. nection of networks that provided worldwide information 


\begin{tabular}{|c|c|c|c|c|c|}
\hline Start & & & & & End \\
\hline Flag & Address & Control & - Informatiōn & FCS & Flag \\
\hline 8 & $8 / 16$ & $8 / 16$ & Variable Length & $16 / 32$ & 8 \\
\hline
\end{tabular}

Figure 6: HDLC Standard / Extended frame format [13]

With ATM, it is not simple to implement things like broadcast or multicast due to its connection-oriented and switched nature. It does not behave the same as a shared-media LAN. This is currently still a problem and attempts at resolving it involve either a revision of the protocols or developing an ATM LAN "emulation." For this reason, ATM will not be further discussed in this paper, since simple use of broadcast and multicast methods is necessary for an autonomous constellation.

\section{IEEE 802.11}

Wireless LANs are becoming more popular in the US and Europe, and as demand for products has grown, standards have been developed to ensure that they are interoperable. The US wireless LAN standard is known as IEEE 802.11 [5], [15].

IEEE 802.11 allows for three different kinds of physical layers, includ-

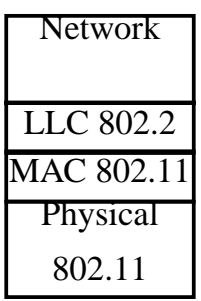

IEEE 802.11 ing direct sequence spread spectrum (DSSS) and frequency hopping spread spectrum (FHSS) which were described earlier. The third kind of physical layer is the infrared. Infrared is not considered here due to range restrictions. It is also seldom used for wireless terrestrial LANs for the same reason.

FHSS breaks up the total bandwidth into frequency channels and takes pseudorandom "hops" from channel to channel after a predetermined time interval has elapsed. For 802.11, the time interval is $<300 \mathrm{~ms}$. As mentioned earlier, this alleviates any collision avoidance issues as the signal is transmitted on any one given frequency only for a very short amount of time. The channel bandwidth is $1 \mathrm{MHz}$, and FHSS avoids repeat use of a channel if at all possible. FHSS uses Gaussian FSK (frequency shift keying) to modulate the signals. FHSS operates in either a $1 \mathrm{Mbps}$ or a $2 \mathrm{Mbps}$ mode.

Instead of dividing the bandwidth into channels, DSSS spreads the signal across the entire bandwidth, which increases bandwidth utilization. The signal modulation is based on PSK (phase shift keying) and is fed to a spreader chip which then multiples the signal with a pseudorandom signal called a chip sequence, which is based on the elevenchip Barker sequence. IEEE 802.11 specifies two data rates for DSSS, 1 Mbps using BPSK (binary PSK) or 2 Mbps using QPSK (quadrature PSK).

In terrestrial systems, DSSS works reliably at greater distances than FHSS $(150 \mathrm{~m}$ vs. $250 \mathrm{~m}$ for a reliable link at $1 \mathrm{Mbps}$ ) but keep in mind these terrestrial transmitters are operating at very low power, for example $30 \mathrm{~mW}$ is used for the 802.11 compatible WaveLAN card. The US 802.11 specifies a maximum RF power level of $1 \mathrm{~W}$. How well 802.11 would scale up to the $10 \mathrm{~km}$ range required by an autonomous constellation has not yet been determined.

The MAC layer for 802.11 has frames with sequence control and retry fields to help minimize interference since the RF components are omnidirectional. The sequence control fields work with type, subtype, duration, and fragmentation fields that are concerned with reliability. Carrier sense multiple access with collision avoidance (CSMA/CA) is used to avoid potential confusion between detecting collisions and noise. The MAC layer also handles acknowledgments in 802.11. Because there is an interframe spacing period of 50 microseconds for all users, the receiver can do a quick 32-bit CRC check and send back an ACK in 10 microseconds, while the medium is still free. The MAC layer also supports "hidden" users that are not within range of their intended recipient but can see someone in between.

IEEE 802.11 uses fragmentation to deal with high RF interference conditions to allow faster sending and receiving. Regular beacons ( every100 milliseconds) are sent to every user in range that includes a timestamp, traffic map, and supported data rates.

IEEE 802.11 has many features that the autonomous constellations could make good use of, but it remains to be determined whether or not IEEE 802.11 can adequately scale up to the desired power and range requirements. The 802.11 standard specifies operation in the ISM unlicensed $2.4 \mathrm{GHz}$ band, in which the FCC limits output power to 1 Watt. The 802.11 MAC protocol can probably be used, but the physical layer would at least need to be adapted to a different frequency and to be compliant in transmitting at higher power levels.

\subsection{CCSDS Lower LaYer Protocols}

\section{CCSDS Proximity-1}




\begin{tabular}{|c|c|c|}
\hline \multirow{3}{*}{ Data Link } & I/O Sublayer & $\begin{array}{l}\text { Interfaces btwn transceiver and on-board data sys- } \\
\text { tem and their applications. Routing, segmentation. }\end{array}$ \\
\hline & Data Services Sublayer & $\begin{array}{l}\text { Defines expedited and sequence controlled data } \\
\text { services like frame ordering and accept/reject. }\end{array}$ \\
\hline & Frame Sublayer & $\begin{array}{l}\text { Frame synchronization, delimiting, FEC and/or } \\
\text { CRC codes. }\end{array}$ \\
\hline \multirow[b]{2}{*}{ Physical } & MAC Sublayer & $\begin{array}{l}\text { Defines how session established, maintained, and } \\
\text { terminated - bridges physical and data link layers. }\end{array}$ \\
\hline & Physical Layer & $\begin{array}{l}\text { Specifications for optimizing link reception and } \\
\text { symbol acquisition. }\end{array}$ \\
\hline
\end{tabular}

Figure 7: The CCSDS Proximity-1 protocol stack [11].

The CCSDS Proximity-1 protocol is based on the CCSDS telecommand frame and is intended for cross-support purposes on proximity links. Proximity links are defined as being short range, bidirectional, fixed or mobile radio links to communicate among landers, rovers, orbiting constellations, and orbiting relays. Proximity links have short time delays, moderate (not weak) signals, and short, independent sessions [11],[16].

With respect to the OSI Model, Proximity-1 (Prox-1) functionality corresponds to the Physical and Data Link layers. However, the Prox-1 data link functionality is broken up into not two, but four sublayers, the frame sublayer, MAC sublayer, data services sublayer, and an Input/Output sublayer as shown in Figure 7.

Prox-1 supports both synchronous and asynchronous modes of communication. For synchronous links, the Prox-1 frame is fixed length, and frames are transmitted continuously for the duration of the session. The fixed length frames are useful in weaker signal environments as FEC block-coding (Reed Solomon) can then be used for the added coding gain. Asynchronous links have variable-length frames, and are intended for use on links with short time delays, moderate signal strength, and short session duration. Prox-1 also uses the virtual channel approach to communication links, however, fixed and variable length frames cannot be multiplexed on the same channel.

Two types of data services are provided - one that accepts and delivers packets, and one that accepts and delivers userdefined data. In the first, packets that are delivered are of a standard format, such as CCSDS source packets, SCPS packets, IP packets, encapsulation packets, etc. In the second, the data transmitted does not have to be recognized by the Prox-1 protocol as a standard packet, but just the user's data.
There are also two grades of service (sequence controlled and expedited) that determine how reliably service data units (SDUs) are sent. One is more connection-oriented, and the other is essentially connectionless. Each grade must be accessed through their own service access point (SAP).

The Sequence Controlled service grade ensures that data is reliably transferred across the space link and delivered in order, without gaps, errors, or duplications within a communication session. Making sure there are no duplications between the termination and initiation of a session is a responsibility that is left to a higher layer. The Sequence Controlled service is based on a go-back-n type of ARQ. The Prox-1 version of an acknowledgment, or "standard report" from the receiving end to the sending end is called a proximity link control word (PLCW).

Expedited service is essentially connectionless and intended for use either with higher level protocols that provide their own retransmission features, or in exigent circumstances such as spacecraft recovery. Expedited SDUs are sent without ARQ, and they are sent independently of Sequence Controlled SDUs. When using expedited service, it is possible to deliver portions of SDUs that are greater than the maximum frame size allowed for the link.

In the most recent version of the CCSDS Red Book for Prox1 , Issue 2 , the physical layer focuses on use on Mars, since its first implementation was on Mars Observer '01. In an upcoming version, there should be an addendum that outlines a physical layer suitable for use on Earth with frequency bands near $26 \mathrm{GHz}$ pending FCC approval. Prox-1 supports many data rates, currently between $2 \mathrm{kbps}$ and $2 \mathrm{Mbps}$. It also allows for convolutional coding (1/2 constraint length 7 Viterbi) for FEC and specifies a link with BER $<10^{-6}$ for both coded and uncoded links. It also allows for Doppler tracking.

The frame sublayer accepts frames from higher layers, adds the PLCW data to complete the frame, forms a status report 
and includes it in the frame, determines the order of transmission, and forms the proximity link transmission units (PLTUs) to be sent. On the receiving end, the frame layer delimits the PLTU, performs FEC or error detection, verifies that it is error free, verifies that it was sent by an acknowledged user, and routes it to a higher layer.

The frame structure includes an attached synchronization marker (ASM) that is 24 bits long when only CRC is used for error detection, and 32 bits long when Reed Solomon FEC is used. Since Reed Solomon codes are block codes, they can only be used with fixed length frames. The Prox-1 32-bit CRC can be used with both fixed and variable length frames.

The MAC sublayer is responsible for establishing, maintaining, and terminating a session. Prox-1 defines away channel contention for single links by using a hailing frequency and a check before allocating channel resources. With multiple links, a collision avoidance approach is taken, where the hailing transmit time is staggered to try to avoid contention.

The data service sublayer exists to control the order of the user data to be transferred, including commands (directives) that are to be transmitted within one session. Expedited service ensures delivery of frames in the order that they are received from a higher layer, but there is no error checking. The data service sublayer is responsible for ensuring the reliability of the sequence controlled data.

The Input/Output sublayer will determine how to integrate received packets into the frames with functions such as segmenting, etc. to interface with the lower sublayer using two queues, one for expedited and one for sequence controlled.

The Prox-1 protocol seems like a very good fit to the needs of the lower layers in an autonomous constellation, which isn't surprising since it was specifically designed for such ISLs. The protocol has not yet become an approved CCSDS standard but is currently in stable Red Book phase. It is the only protocol being considered for use by spacecraft involved in the Mars Network, and is the primary protocol being considered by the New Millennium Program ST5 constellation, which is discussed in Section 5.

\section{CCSDS SCPS}

The SCPS protocol stack is the "space equivalent" of TCP/IP and was designed with the goal of extending internet connectivity into space. In addition to the error-protected, sequenced data streams with real time acquisition and quick look analysis of the standard CCSDS protocols, it also supports automatic, real-time retransmission to provide complete and best-effort data streams and reliable file transfer. The SCPS protocol stack begins at the network layer, as does TCP/IP, so although it remains a contender as a higher layer protocol, it is not a complete lower layer protocol. The SCPS Network Protocol (SCPS-NP) went CCSDS Blue Book in
May 1999, and as mentioned in the previous section, can run over Prox-1 [17].

\section{CCSDS AOS}

CCSDS extended its previous space/ground and ground/ space link recommendations to reflect the needs of the Advanced Orbiting Systems (AOS) of the 1990s and beyond, providing a more diverse and flexible set of data handling services. These services are intended for uses such as manned and man-tended space stations, unmanned space platforms, free-flying spacecraft, and any other spacecraft needing services to concurrently transmit multiple digital data types such as audio and video. However, the AOS protocols are not intended for space-space links, as the Prox-1 protocol is [18].

\subsection{Autonomous Constellations AND ISLS}

\section{New Millennium Program ST5}

NASA's Space Technology 5 (ST5) mission, called "The Nanosat Constellation Trailblazer" is the fourth deep space mission in NASA's New Millennium Program. ST5 is slated as a secondary launch in 2003 and plans to fly a constellation of three nanosatellites $(21.5 \mathrm{~kg}$ each) at about a $200 \mathrm{~km}$ by $36,000 \mathrm{~km}$ altitude to monitor the magnetosphere.

Like TechSat 21, the spacecraft will be used to test the "virtual satellite" concept of operating a constellation as a single system. The ST5 satellites will attempt to perform coordinated movements, communication, and scientific observations of the magnetosphere as if they were a single larger spacecraft. This includes the goal of having the spacecraft autonomously stay in contact with each other, share information, and reconfiguring onboard instruments and systems to behave as a single unit. The mission is managed by NASA's Goddard Space Flight Center (GSFC) in Greenbelt, Maryland.

JPL is working on the miniature spacecraft communications system that provides the capability to communicate between spacecraft and determine the positions of spacecraft relative to each other and the ground using GPS, which is very similar to the TechSat 21 ISL communications approach. The data rate for ST5 will be lower, however, because it does not currently include transferring a great amount of payload data. For a scenario where data is transferred in order to do parallel processing using constellation resources, the data rate would significantly increase.

With respect to a lower layer protocol selection for this project, sources at JPL report that they started out not considering any options other than the CCSDS Proximity-1 specification. This was chosen due to some similar work being done at JPL on the Mars Network cross-links where Proximity-1 is required. They have recently started consider- 


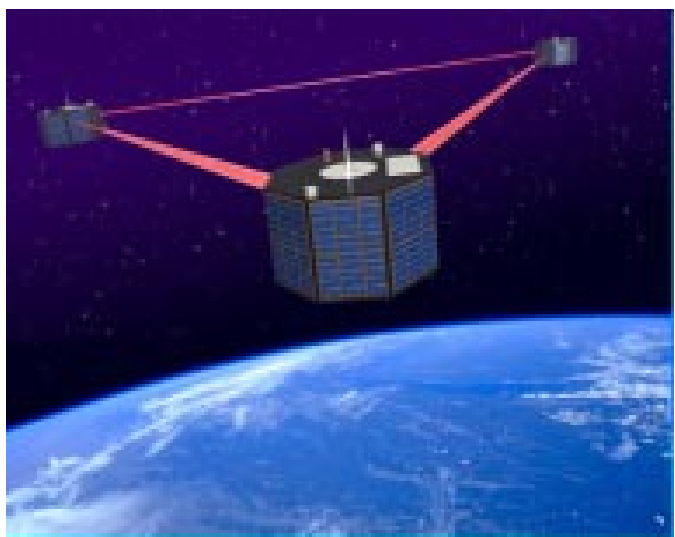

Figure 8: Artist's conception of ST5

ing using the MAC layer of IEEE 802.11, but have not as of the writing of this paper done a thorough evaluation on it[19].

The data rate that ST5 will be using is low, only about $1 \mathrm{kbps}$ at the moment, and they are looking at using the $\mathrm{S}$ frequency band. The spacecraft are power limited, with the transceiver at less than $10 \mathrm{~W}$, including the baseband processor and RF power electronics. The maximum ranges they are designing to vary between 100 to $10,000 \mathrm{~km}$, depending on mission configuration and life-cycle.

\section{SSTL SNAP-1 and Tsinghua-1}

The Surrey Nanosatellite Applications Platform (SNAP) is a flexible commercial $6.5 \mathrm{~kg}$ nanosatellite platform aimed at providing access to space at a reasonable cost. SNAP functionality includes formation flying, inter-spacecraft communications, on-board navigation, propulsion, and machine vision for remote inspection. The Tsinghua-1 microsatellite is a joint venture between Tsinghua University in China and SSTL. Tsinghua-1 carries a camera capable of 39 meter resolution images in three spectral bands and is designed to be a prototype for a future Disaster Monitoring Constellation (DMC) proposed by SSTL, a network of five small satellites to monitor natural and man-made disasters.

Both SNAP-1 and Tsinghua-1 were launched from the Plesetsk Cosmodrome into a $650 \mathrm{~km}$ sun-synchronous orbit on June 28, 2000. The recent update is that most of the systems have already been tested successfully, although there is no itemized list currently available, and it is not known whether the ISL has yet been tested [7].

The primary goals of the SNAP-1 mission included demonstrating an intersatellite communication channel between the two satellites, experimenting with GPS ranging between the two satellites, and demonstrating formation flying. SNAP-1 is currently doing earth observing with four sub-miniature CMOS cameras.
The SNAP-1 and Tsinghua-1 ISLs are RF, with a 9.6kbps data rate. SNAP-1 uses an HDLC controller implemented in a FPGA for communication at close range, as well as for the synchronous uplink and downlink. The electrical power consumption of the ISL RF system is on the order of $400 \mathrm{~mW}$. There is currently no goal for SNAP-1 to communicate with any other spacecraft than Tsinghua-1. The GPS ranging on SNAP-1 will be accurate only to about 15 meters.

\section{TechSat 21}

As described earlier, TechSat 21 is the autonomous forma-

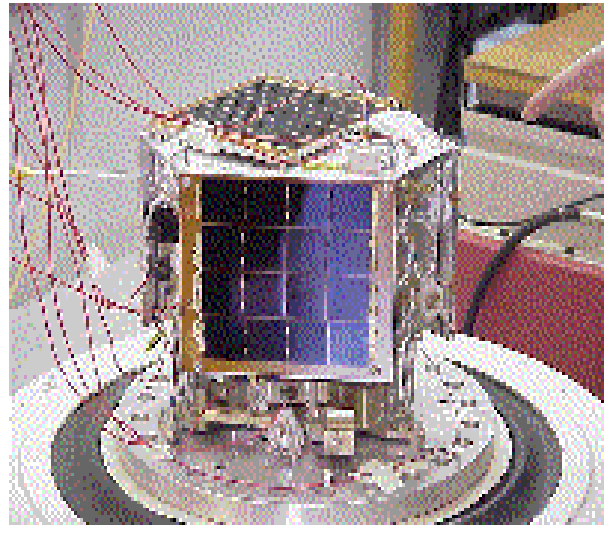

Figure 9: The Surrey SNAP-1 satellite.

tion-flying constellation being developed by AFOSR for remote sensing applications and currently has a test flight demo scheduled for 2003 and plans to have an operational cluster by 2005 . The microsatellites will be in close proximity clusters, with the possibility of 40 clusters in orbit at a time. One of the goals of this program is to be able to easily interchange single satellites and thus be able to vary the capabilities of the cluster[20].

\subsection{SUMmarY}

\section{Recap}

First, a description of ISL functionality was given, and it was shown that the ISL designs for current GEO and LEO broadband or mobile communications networks are not similar enough to the requirements for an autonomous formationflying constellation that their lower layer protocols be considered for comparison. This was followed by a brief overview of the networking principles necessary to compare lower layer protocols against each other. Then, a detailed summary of existing and upcoming protocol standards were presented:

It was concluded that ATM did not adequately support multiple access, TCP/IP and SCPS were too high up the protocol stack to be considered as a lower layer protocol, AOS was not intended for space-space links, and that the IEEE 802.11 physical layer would need to be entirely revamped to meet 
physical layer requirements. Both X.25/LAP-B and CCSDSProximity-1 remained as possible options for the ISL lower layer protocol, and the possibility of using the IEEE 802.11 MAC layer was also acknowledged.

Three similar missions that have been or are being designed were described. The requirements of the NMP ST5 spacecraft more closely match those of TechSat 21 in terms of power, range, and multiple access. However, since all three programs have come to the conclusion that either some version of HDLC (SNAP-1) or CCSDS Proximity-1 (ST5) should be used, this paper will conclude with a comparison of the two.

\section{X.25/LAP-B vs. CCSDS Prox-1}

It is evident that Proximity-1 was designed specifically for close-range space-space links, where X.25 was created almost 30 years ago with terrestrial networks in mind. However, there are existing commercial parts, experience, and support for an X.25 or HDLC system where there are no commercial parts or support for Prox-1 yet available, although that should change as Prox-1 becomes an approved CCSDS standard (blue book). There have been recent talks with NASA GSFC about manufacturing chips, and Prox-1 has been implemented already on the Mars Surveyor 2001 Orbiter. Prox-1 has also been baselined by ESA for the Mars Express MARESS transceiver and Beagle II lander for their 2003 mission. However, commercial parts are not the same as specific flight hardware, which would probably still need to be procured in either case.

The HDLC-based X.25 protocol depends on a specific eightbit sequence to determine the start and end of a frame, and to ensure it is not repeated, it uses the technique of bit-stuffing on the rest of the data. This could be problematic given that in low SNR environments, cycle slips can occur at the receiver, which show up as one or more bit slips in the data. This could cause an HDLC frame to be interpreted as two separate shorter frames or a long frame could be split into two shorter frames. This should not happen with Proximity1 , as there is an attached synchronization marker (ASM) that is either 24 or 32 bits long, depending whether block coding is used or not. This allows for more reliable synchronization and advance knowledge of frame length, as the probability of error in the frame length field is very low.

Recall that HDLC uses a 16-bit CRC check called CRCCCITT, and from the discussion of CRC checks that this CRC can protect against all single, double, and odd bit errors plus burst layers that are shorter than the degree of the polynomial, which is 16 in this case, provided no other errors occur within the frame. Prox-1's CRC may be able to protect up to 32 bit long burst lengths. The FEC option that Prox-1 provides can correct still more errors, possibly up to multiple packet errors. However, a longer CRC or use of FEC increases the amount of overhead.
Prox-1 offers two grades of service, one with fixed length frames and the other expedited with variable length frames. All HDLC frames are variable length. This means that no block coding can be used with FEC in HDLC to reduce the bit error rate. If there are ranging requirements, for example that satellite position information be sent with a bit error rate less than $10^{-12}$, then high performance block codes such as the Reed-Solomon codes would be beneficial in attempting to achieve this, especially at greater distances with lower SNR.

HDLC uses modes - it has three operational modes, the mode of choice for ISLs being ABM. In addition it has three non-operational modes for disconnecting and initialization. Proximity-1 is modeless, telemetry, command and ranging/ timing services can all take place concurrently without scheduling or switching modes by mission operations. Prox1 was also designed with the realization that the forward and return links may not be symmetrical, where HDLC was designed for symmetric links.

Added bonuses for Prox-1 include the fact that since Prox-1 can carry CCSDS frames in its packets, it should be possible to communicate directly with a CCSDS ground station as backup. Also, if it is desirable at some point to talk to other autonomous spacecraft near to the constellation, it would be prudent to choose a protocol that other agencies are likely to use. CCSDS is prevalent in ground/space communications, and Prox-1 will be a CCSDS standard that is intended for space-space communication and supported by both national and international agencies. However, Prox-1 is currently still in Red Book stage and HDLC has decades of commercial production and existing engineering expertise, even though it was not intended for use on intersatellite links. An experimental comparison of two similar implemented systems including a cost estimate is needed to determine whether the benefits built into Prox-1 will outweigh the availability of existing standards such as HDLC or IEEE 802.11.

\subsection{REFERENCES}

[1] Jacobs, I.M., Binder, R., Hoversten E.V. "General Purpose Packet Satellite Networks," Proceedings of the IEEE, vol. 66, no. 11, pp. 1448-1467, 1978.

[2] Bertsekas, D. and Gallager, R. Data Networks, 2nd edition, Prentice Hall, 1992.

[3] Maral, G., Bousquet, M. Satellite Communications Systems, 3rd edition, Wiley, 1998.

[4] Ziemer, R.E and Tranter, W.H. Principles of Communications, Systems, Modulation and Noise, 3rd edition, Boston: Houghton Mifflin, 1990. 
TABLE 3: Basic Requirements for Formation Flying Constellations

\begin{tabular}{||l|lll||}
\hline \hline ISL: & TechSat 21 & NMP ST5 & SSTL SNAP-1 \\
\hline \hline Power & $<15 \mathrm{~W}$ & $<10 \mathrm{~W}$ & $\sim 400 \mathrm{~mW}$ \\
Range & $\sim 10 \mathrm{~km}$ & $100-10,000 \mathrm{~km}$ & $?$ \\
Data Rate & $0.1-2 \mathrm{Mbps}$ & $\sim 1 \mathrm{kbps}$ & $9.6 \mathrm{kbps}$ \\
Band & Ku-band? & S-band & S-band \\
S/C Mass & $\sim 120 \mathrm{~kg}$ Micro & $\sim 21.5 \mathrm{~kg}$ Nano & $6.5 \mathrm{~kg}$ Nano \\
Multi Access? & Yes & Yes & No \\
Altitude & $700 \mathrm{~km}$ & $200-35,000 \mathrm{~km}$ & $650 \mathrm{~km}$ \\
Protocol & $?$ & Prox-1 & HDLC \\
\hline \hline
\end{tabular}

[5] Welch, J. (1999) "Wireless Networking and the AirPort, IEEE 802.11”, MacTech vol. 15, no. 12, pp. 14-32, December 1999.

[6] Wood, L. Correspondence with Lloyd Wood at University of Surrey regarding existing LEO/GEO constellations and SNAP-1, June-July, 2000.

[7] Salvignol, J. Correspondence with Jerome Salvignol at Surrey Satellite Technology Limited, July 21, 2000.

[8] Peterson, L., Davie, B. (1996) Computer Networks, a Systems Approach, Morgan Kaufmann.

[9] Halsall, F. (1996) Data Communications, Computer Networks, and Open Systems, 4th edition, Addison Wesley.

[10] Information Technology - Open Systems Interconnection - Basic Reference Model: The Basic Model. International Standard, ISO/IEC 7498-1. 2nd ed. Geneva: ISO 1994.

11] Proximity-1 Space Link Protocol, Recommendation for Space Data System Standards, CCSDS 211.0-R-2. Red Book. Issue 2. Washington, D.C., CCSDS, January 2000.

[12] X.25 Recommendation (10/96) Interface between Data Terminal Equipment (DTE) and Data Circuit-terminating Equipment (DCE) for terminals operating in the packet mode and connected to public data networks by dedicated circuit, ITU-T.

[13] Information Technology - Telecommunications and information exchange between systems - High level data link control (HDLC) procedures - Elements of procedures. International Standard, ISO/IEC 4335-1. 5th ed. Geneva: ISO 1993.

[14] Murhammer, M. et al. (1989) TCP/IP Tutorial and Technical Overview, 6th edition, Prentice Hall.

[15] Lough, D., Blankenship, K., Krizman, K. (1997) A Short Tutorial on Wireless LANs and IEEE 802.11, Virginia Polytechnic Institute and State University.

[16] Kazz, G. Correspondence with Greg Kazz at JPL regarding CCSDS Proximity-1, July 10, 2000.

[17] Space Communications Protocol Specification (SCPS) Network Protocol (SCPS-NP), Recommendation for Space Data System Standards, CCSDS 713.0-B-1. Blue Book. Issue 1. Washington, D.C., CCSDS, May 1999. Adopted as ISO/DIS 15893.

[18] Advanced Orbiting Systems, Networks and Data Links: Architectural Specification, Recommendation for Space Data System Standards, CCSDS 701.0-B-2. Blue Book. Issue 1. Washington, D.C., CCSDS, November 1992, Reconfirmed June 1998. Adopted as ISO 13420:1997.

[19] Farrington, A. Correspondence with Allen Farrington at JPL regarding NMP ST5, July 17, 2000.

[20] Zetocha, Paul (2000) TechSat-21 Command, Control, and Communications Planning Document, Version 1.03, June 6, 2000. Draft. 